\title{
Feasibility of a Microarray-Based Point-of-Care CYP2C19 Genotyping Test for Predicting Clopidogrel On-Treatment Platelet Reactivity
}

\author{
Hyojin Chae, ${ }^{1,2}$ Myungshin Kim, ${ }^{1,2}$ Yoon-Seok Koh, ${ }^{3}$ Byung-Hee Hwang, \\ Min-Kyu Kang, ${ }^{4}$ Yonggoo Kim, ${ }^{1,2}$ Hae-il Park, ${ }^{1,2}$ and Kiyuk Chang ${ }^{4}$ \\ ${ }^{1}$ Department of Laboratory Medicine, Bucheon St. Mary's Hospital, 2 Sosa-dong, Wonmi-gu, Gyeonggi-do, \\ Bucheon-si 420-717, Republic of Korea \\ ${ }^{2}$ Catholic Laboratory Development and Evaluation Center, College of Medicine, The Catholic University of Korea, \\ Seoul 137-701, Republic of Korea \\ ${ }^{3}$ Cardiovascular Center and Cardiology Division, Uijeongbu St. Mary's Hospital, Uijeongbu 480-717, Republic of Korea \\ ${ }^{4}$ Cardiovascular Center and Cardiology Division, Seoul St. Mary's Hospital, 505 Banpo-dong, Seocho-gu, \\ Seoul 137-701, Republic of Korea
}

Correspondence should be addressed to Hae-il Park; phi@catholic.ac.kr and Kiyuk Chang; kiyuk@catholic.ac.kr

Received 17 August 2012; Accepted 11 March 2013

Academic Editor: Yasemin Alanay

Copyright (C) 2013 Hyojin Chae et al. This is an open access article distributed under the Creative Commons Attribution License, which permits unrestricted use, distribution, and reproduction in any medium, provided the original work is properly cited.

\begin{abstract}
Clopidogrel is a prodrug which is converted into active metabolite by cytochrome P450 isoenzyme, CYP2C19. Numerous polymorphisms of CYP2C19 are reported, and a strong link exists between loss-of-function (LOF) or gain-of-function polymorphisms, clopidogrel metabolism, and clinical outcome. Hence, a fully automated point-of-care CYP2C19 genotyping assay is more likely to bring personalized antiplatelet therapy into real practice. We assessed the feasibility of the Verigene 2C19/CBS Nucleic Acid Test, a fully automated microarray-based assay, compared to bidirectional sequencing, and performed VerifyNow P2Y12 assay to evaluate the effect of CYP2C19 polymorphisms on on-treatment platelet reactivity in 57 Korean patients treated with clopidogrel after percutaneous coronary intervention. The Verigene $2 \mathrm{C} 19$ /CBS assay identified ${ }^{*} 2,{ }^{*} 3$, and ${ }^{*} 17$ polymorphisms with $100 \%$ concordance to bidirectional sequencing in 180 minutes with little hands-on time. Patients were classified into 4 groups: extensive $\left({ }^{*} 1 /{ }^{*} 1 ; n=12\right.$, $21.1 \%)$, intermediate $\left({ }^{*} 1 /{ }^{*} 2,{ }^{*} 1 /{ }^{*} 3 ; n=33,57.9 \%\right)$, poor $\left({ }^{*} 2 /^{*} 2,{ }^{*} 2 /{ }^{*} 3\right.$, and $\left.{ }^{*} 3 /{ }^{*} 3 ; n=11,19.3 \%\right)$, and ultrarapid metabolizers $\left({ }^{*} 1{ }^{*} 17\right.$; $n=1,1.8 \%)$. The prevalence of the $\mathrm{CYP} 2 \mathrm{C}_{1}{ }^{*} 2,{ }^{*} 3$, and ${ }^{*} 17$ alleles was $36.0 \%, 12.3 \%$, and $0.9 \%$. Platelet reactivity showed gene dose response according to the number of CYP2C19 LOF allele. In conclusion, the Verigene 2C19/CBS assay gave accurate CYP2C19 genotype results which were in well match with the differing on-treatment platelet reactivity.
\end{abstract}

\section{Introduction}

Clopidogrel is a thienopyridine prodrug, whose active liver metabolite covalently binds cysteine residues of the platelet surface P2Y12 receptor, irreversibly blocking the receptor, leading to inhibition of platelet aggregation in response to $\mathrm{ADP}$ and also to other agents through the inhibitory effect on released ADP.

The conversion of clopidogrel to its active metabolite relies on the CYP2C19 enzyme, a member of the hepatic cytochrome P450 family. Numerous polymorphisms in CYP2C19 have been identified and individuals can be classified as the phenotype of extensive metabolizers (EMs), intermediate metabolizers (IMs), poor metabolizers (PMs), and ultrarapid metabolizers (UMs) according to the polymorphism of CYP2C19. Among the PM phenotypes, CYP2C19 ${ }^{*} 2$ and ${ }^{*} 3$ polymorphisms are the most frequent and both alleles confer loss-of-function (LOF) leading to a complete loss of the enzyme activity. Indeed, in patients who carry the CYP2C19 ${ }^{*} 2$ or ${ }^{*} 3$ allele, the conversion of clopidogrel to its active metabolite is reduced, resulting in decreased response of platelets to clopidogrel treatment and worse cardiovascular outcome. 
Clopidogrel on top of aspirin has revolutionized the treatment of patients with acute coronary syndrome and undergoing percutaneous coronary intervention (PCI) $[1,2]$. However, interindividual variation of platelet inhibition by clopidogrel has been noted, and from $5 \%$ to $11 \%$ of patients on clopidogrel treatment experience acute or subacute thrombosis after a coronary event or implantation of a coronary stent [1]. Accordingly, a black-box warning was added to the clopidogrel package insert indicating a significant clinical link between CYP2C19 LOF genotypes $\left({ }^{*} 2\right.$ and ${ }^{*} 3$ ) and poor metabolism of clopidogrel [3]. Therefore, a CYP2C19 genotyping assay with a rapid sample-to-result time could be beneficial in the appropriate dosing of clopidogrel based on the genotype of the patient and/or permitting change to other antiplatelet agents in a timely manner.

The aim of this study was to assess the performance of the Verigene 2C19/CBS Nucleic Acid Test (Nanosphere, Northbrook, IL, USA), a fully automated microarray-based assay that identifies 12 allelic variants of CYP2C19 $\left({ }^{*} 1-^{*} 10\right.$, ${ }^{*} 13$, and $\left.{ }^{*} 17\right)$ in a rapid turnaround time of approximately 3 hours [3], and to study the influence of the CYP2C19 allelic variants derived from the Verigene test on on-treatment platelet reactivity as assessed by VerifyNow P2Y12 assay (Accumetrics, San Diego, CA, USA) in Korean patients treated with clopidogrel after PCI with the use of drug-eluting stents (DESs).

\section{Materials and Methods}

2.1. Patients. Peripheral blood samples were collected from 57 consecutive patients subjected to PCI. All patients were preliminarily treated with $100 \mathrm{mg} /$ day of aspirin followed by coadministration of clopidogrel (loading dose, $600 \mathrm{mg}$; maintenance dose, $75 \mathrm{mg} /$ day). Exclusion criteria were platelet count outside from 100 to $450 \times 10^{9} /$ L range; hematocrit $<25 \%$ or hemoglobin $<8 \mathrm{~g} / \mathrm{dL}$; and chronic renal failure requiring dialysis. The study was conducted in accordance with the Declaration of Helsinki ethical guidelines and was approved by the Institutional Review Board at Catholic Medical Center.

2.2. Platelet Function Testing with VerifyNow P2Y12 Assay. The blood was drawn from the antecubital vein at $48 \mathrm{~h}$ after clopidogrel loading dose into a $3.2 \%$ sodium citrate tube for the VerifyNow P2Y12 assay. The time interval between blood sampling and VerifyNow P2Y12 testing did not exceed 2 hours. Platelet reactivity was assessed by the VerifyNow P2Y12 assay, and the VerifyNow P2Y12 assay was performed as previously described [4]. With this assay, higher P2Y12 reaction units (PRU) reflect greater ADP-mediated platelet reactivity. Cut-off value for high on-treatment residual ADPinducible platelet reactivity (HRPR) was PRU > 235 for the VerifyNow P2Y12 assay according to the published consensus statement [5].

2.3. Genotyping with Verigene 2C19/CBS Nucleic Acid Test. The blood was drawn from the antecubital vein into an EDTA tube for genotyping. For the Verigene 2C19/CBS Nucleic Acid Testing, the EDTA-anticoagulated whole blood (EDTA-WB) samples could be stored at $2-8^{\circ} \mathrm{C}$ for up to 10 days before processing. Briefly, a single-use extraction tray containing all necessary reagents to lyse, extract, and purify DNA from WB was loaded into the Verigene Processor SP (Nanosphere). $1.0 \mathrm{~mL}$ of EDTA-WB was transferred to the sample well in the extraction tray. A single-use test cartridge containing the slide array and hybridization reagents was loaded into the Verigene Processor SP, and the assay was started. On completion of the assay, the test cartridge was removed from the processor, and the hybridization slide was inserted into the Verigene Reader.

2.4. Direct Sequencing. To evaluate the accuracy of the genotype results obtained with the Verigene 2C19/CBS Nucleic Acid Test we performed Sanger-based direct sequencing method in parallel. Briefly, the Genomic DNA was isolated from the peripheral leukocytes using the QIAmp DNA Mini Kit (Qiagen, Hamburg, Germany). PCR was carried out using previously published primer sets for ${ }^{*} 2$ and ${ }^{*} 3$ [6] and a newly designed primer set for ${ }^{*} 17$. The PCR amplicons were sequenced using the Big Dye terminator v3.1 cycle sequencing kit (Applied Biosystems, Foster City, CA, USA) on an ABI PRISM 3100 Genetic Analyzer (Applied Biosystems). The chromatograms were analyzed with the Sequencher software version 4.9 (Gene Codes).

2.5. Statistical Analysis. PRU values of VerifyNow P2Y12 assay were compared between the metabolizer statuses by one-way analysis of variance (ANOVA). MedCalc version 12.1.4 (Mariakerke, Belgium) was used for all statistical analyses; $P<0.05$ was considered statistically significant.

\section{Results}

Clinical and laboratory characteristics of the total population are presented in Table 1. The average age was 67.0 years and about $65 \%$ of patients were men. According to the defined cut-off value, the frequency of clopidogrel resistance was $42.1 \%$. Baseline demographics, clinical presentation, and treatment were well balanced between the CYP2C19 LOF carrier/and noncarrier groups $(P>0.05)$.

3.1. Verigene 2C19/CBS Nucleic Acid Test for Identifying CYP2C19 Polymorphisms. An initial result was obtained for 53 of 57 specimens (93.0\%) using the Verigene 2C19/CBS Nucleic Acid Test. On retesting, all 4 samples gave a definitive result. The total time-to-result was approximately 3 hours with about 15 minutes of hands-on time. The comparison of polymorphism results between bidirectional sequencing and the Verigene 2C19/CBS Nucleic Acid Test revealed 100\% concordance rate for all 57 specimens that were analyzed.

3.2. Genotype Frequencies and Classification of Metabolizer Statuses. Distributions of the CYP2C19 alleles, genotypes, and the predicted phenotypes in our study population are given in Table 2. The frequency of the LOF genotype was high $(77 \%)$. The prevalence of the gain-of-function variant, CYP2C19 * 17 allele, was low (1\%), and the prevalence of the CYP2C19 ${ }^{*} 2$ and ${ }^{*} 3$ alleles were $36 \%$ and $12 \%$, respectively. Of the 57 patients included in the study, $12(21 \%)$ were classified as extensive (EM), 1 (2\%) as ultrarapid (UM), $33(58 \%)$ as intermediate (IM), and 11 (19\%) as poor (PM) metabolizers. 
TABLE 1: Baseline characteristics of the study population.

\begin{tabular}{lcccc}
\hline & Overall & LOF & No LOF & \multirow{2}{*}{ P value } \\
& $N=56$ & $N=44$ & $N=12$ & \\
\hline Age & 67.0 & 67.0 & 66.0 & 0.7 \\
Male gender & $36(64.3)$ & $29(65.9)$ & $7(58.3)$ & 0.7 \\
Smoking & $10(17.9)$ & $7(15.9)$ & $3(25.0)$ & 0.4 \\
Hypertension & $33(58.9)$ & $25(56.8)$ & $8(66.7)$ & 0.7 \\
Diabetes mellitus & $15(26.8)$ & $11(25.0)$ & $4(33.3)$ & 0.7 \\
Dyslipidemia & $12(21.4)$ & $9(20.5)$ & $3(25.0)$ & 0.7 \\
Prior MI & $3(5.4)$ & $1(2.3)$ & $2(16.7)$ & 0.1 \\
Prior PCI & $11(19.6)$ & $7(15.9)$ & $4(33.3)$ & 0.2 \\
BMI & 24.8 & 26.1 & 24.3 & 0.2 \\
Statin & $15(26.8)$ & $13(29.5)$ & $2(16.7)$ & 0.5 \\
$\beta$-blocker & $9(16.1)$ & $5(11.4)$ & $4(33.3)$ & 0.09 \\
ACE inhibitor & $4(7.1)$ & $3(6.8)$ & $1(8.3)$ & 1 \\
CCBs & $11(19.6)$ & $8(18.2)$ & $3(25.0)$ & 0.7 \\
PPIs & $2(3.6)$ & $1(2.3)$ & $1(8.3)$ & 0.4 \\
Platelet count & 215.5 & 221.0 & 215.5 & 1 \\
C-reactive protein & 0.1 & 0.1 & 0.1 & 0.2 \\
\hline
\end{tabular}

The denotations are LOF: loss-of-function; MI: myocardial infarction; PCI: percutaneous coronary intervention; BMI: body mass index; ACE: angiotensin converting enzyme inhibitor; CCB: calcium channel blocker; and PPI: proton pump inhibitor.

3.3. Influence of Metabolizer Statuses on Platelet Reactivity. Platelet reactivity measured by the VerifyNow P2Y12 assay differed significantly according to metabolizer statuses when tested by analysis of variance (Figure 1). Higher onclopidogrel platelet reactivity was observed as the number of CYP2C19 LOF allele increased (UM 4.0; EM, 177.7; IM, 201.7; and PM 277.0). Student-Newman-Keuls test for pairwise comparison revealed a significant difference between UM and IM/PM using the VerifyNow P2Y12 assay.

\section{Discussion}

In this study, we found that the test reliability of the Verigene 2C19/CBS Nucleic Acid Test for the identification of CYP2C19 polymorphisms was $100 \%$ accurate as compared with the bidirectional sequencing method. In addition, this Verigene test offered rapid detection time and enabled point-of-care diagnosis without conventional DNA extraction, PCR steps, and sequencing. Genotyping assays for CYP2C19 can be performed with numerous molecular methods, such as real-time PCR, allele-specific PCR, PCRRFLP, pyrosequencing, and bidirectional sequencing. Also a growing number of commercial analytical platforms are available, and these include the INFINITI (AutoGenomics, Inc., Vista, Carlsbad, CA, USA) [7], the Verigene, eSensor XT8 (Genmark, Carlsbad, CA, USA) [8], Spartan RX (Spartan Biosciences, Ottawa, ON, Canada) [9], Invader (Hologic, Bedford, MA, USA) [10], and Luminex assays (Luminex, Austin, TX, USA) [8]. Although the comparison of different genotyping methods is beyond the scope of this paper, the Verigene assay utilizes signal amplification and not target amplification, and therefore it has a unique advantage of

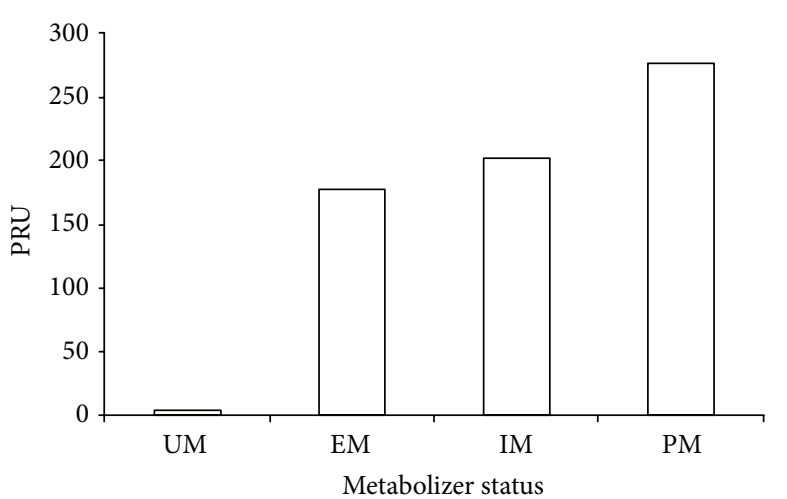

FIGURE 1: Result from the VerifyNow P2Y12 assay according to CYP2C19 metabolizer statuses. Data are shown as mean. The denotations are PRY: P2Y12 reaction units; UM: ultrarapid; EM: extensive; IM: intermediate; and PM: poor metabolizer.

operating in a PCR-free environment. Also Verigene has the shortest TATs among commercial platforms that are capable of genotyping a certain number of polymorphisms and also the least complex to operate. And therefore the assay can be readily implemented in clinical laboratories without extensive experience in molecular techniques. The limitation of the Verigene assay is the no call rate of $7-10 \%$. The rate of no call at first attempt was $7.3 \%$ in our study; however, in our experience, the no call errors were resolved with repeat testing.

The inhibitory effect of clopidogrel on platelet function shows marked interindividual variability. The prevalence of clopidogrel nonresponsiveness has been reported to be from $4 \%$ to $30 \%$ in patients with coronary artery disease [11]. Several factors including underdosing, inappropriate dosing, variable absorption of the prodrug, variable clearance of the active metabolite, potential drug-drug interactions, P2Y12 receptor variability, and genetic polymorphisms of cytochrome P450 isoenzymes are possible mechanisms of clopidogrel resistance [11]. In this respect, CYP2C19 polymorphisms specifically addresses the issues associated with dosing, since in vivo transformation of the prodrug to its active metabolite is dependent on the hepatic cytochrome $\mathrm{P} 450$ isoenzyme CYP2C19. Today, at least 25 single nucleotide polymorphisms (SNPs) in the gene coding for CYP2C19 have been described. Of these, $C Y P 2 C 19^{*} 2,{ }^{*} 3$ are the most frequent LOF polymorphisms and thus the main genetic determinants of clopidogrel response variability. In this study, we also observed that CYP2C19 LOF alleles were significantly associated with reduced antiplatelet efficacy of clopidogrel.

There exists a marked interracial difference in the frequency of the CYP2C19 polymorphisms. Asians have a higher prevalence of CYP2C19 LOF alleles and PM phenotypes (from $13 \%$ to $23 \%$ in Asians and from $1 \%$ to $6 \%$ in Caucasians) $[12,13]$. In this study, the prevalence of CYP2C19 PM, ${ }^{*} 2$, and ${ }^{*} 3$ alleles were $19.3 \%, 36.0 \%$, and $12.3 \%$, respectively. These results are in agreement with the observed allele frequencies of $28.6 \%$ and $7.4 \%$ for CYP2C19 ${ }^{*} 2$ and ${ }^{*} 3$ alleles in a previous report of 200 Korean individuals [14]. The carriage prevalence of the CYP2C19 LOF variant is $77.2 \%$ in this study, which is 
TABLE 2: Distributions of the CYP2C19 alleles, genotypes, and the predicted phenotypes.

\begin{tabular}{|c|c|c|c|c|c|}
\hline Allele & Frequency, $n(\%)$ & Genotype & Frequency, $n(\%)$ & Phenotype & Metabolizer status \\
\hline${ }^{*} 1$ & $58(51)$ & ${ }^{*} 17 / \mathrm{wt}$ & $1(2)$ & Rapid heterozygous & UM \\
\hline$* 2$ & $41(36)$ & $\mathrm{wt} / \mathrm{wt}$ & $12(21)$ & Extensive & EM \\
\hline *3 & $14(12)$ & ${ }^{*} 2 / \mathrm{wt}$ & $24(42)$ & Intermediate & IM \\
\hline \multirow[t]{3}{*}{${ }^{*} 17$} & $1(1)$ & $* 3 /$ wt & $9(16)$ & Intermediate & IM \\
\hline & & ${ }^{*} 2 /^{*} 2$ & $6(10)$ & Poor & PM \\
\hline & & ${ }^{*} 2 /{ }^{*} 3$ & $5(9)$ & Poor & PM \\
\hline
\end{tabular}

The denotations are wt: wild type; UM: ultrarapid metabolizer; EM: extensive metabolizer; IM: intermediate metabolizer; and PM: poor metabolizer.

also in line with the reported prevalence of $55 \%$ to $70 \%$ among Asians [12].

Multiple tests are available for the monitoring of antiplatelet therapy, especially aspirin and clopidogrel. Light transmission aggregometry (LTA) is the gold standard for assessing the platelet response to ADP [4], but this method is laborious and weakly standardized. In the present study, the VerifyNow P2Y12 assay, a method that shows the strongest correlation with the LTA among whole blood-based methods, was used to assess clopidogrel-mediated platelet inhibition. Platelet reactivity measured by the VerifyNow P2Y12 assay significantly differed according to metabolizer statuses when tested by analysis of variance in our population. Higher onclopidogrel platelet reactivity was observed as the number of CYP2C19 LOF allele increased (UM 4.0; EM, 177.7; IM, 201.7; and PM 277.0), but post hoc analysis did not reach statistical significance for linear trend, probably as a result of the small number of patients. Interestingly, a rare but well-recognized gain of function allelic variant CYP2C19 * 17 was identified in our study. Student-Newman-Keuls test for pairwise comparison revealed a significant difference between UM and both IM/PM platelets reactivity using the VerifyNow P2Y12 assay.

The Verigene 2C19/CBS Nucleic Acid Test is a fully automated microarray test that utilizes gold nanoparticle-conjugated oligonucleotide probes to detect nucleic acids captured by array probes, and this method eliminates the need for target amplification, namely, a PCR step, before array hybridization $[3,15]$. Therefore the assay is less prone to errors introduced during the conventional nucleic acid extraction and target amplification processes and has a rapid turnaround-time. The initial call rate of this study was $93.0 \%$ which is similar to the reported initial call rate of $93.5 \%$ in a previous study [3], and the assay identified heterozygous and homozygous ${ }^{*} 2,{ }^{*} 3$, and ${ }^{*} 17$ polymorphisms with $100 \%$ concordance to bidirectional sequence analysis in 57 patient samples. Most importantly, the total time to result was approximately 3 hours with less than 15 minutes of hands-on time.

There are a number of limitations of this study. Our cohort consisted of only 57 patient samples and the small sample size related to lack of statistical power. Also another limitation of our study was the use of PRU values as measured by VerifyNow P2Y12 assay as a measure of clinical efficacy and did not include clinical endpoints. However, the analytical validation of Verigene assay as well as clinical validation using
PRU values as surrogate endpoints to clinical efficacy serves a crucial role in providing the link of the point-of-care microarray CYP2C19 genotyping assay towards pharmacogenetic dosing of clopidogrel in real clinical practice.

\section{Conclusion}

In conclusion, the concordance rate of the Verigene 2C19/ CBS Nucleic Acid Test with Sanger's sequencing method was $100 \%$ in this study of Korean patients treated with clopidogrel after coronary stenting. In addition, the genetic test results of the CYP2C19 polymorphisms highly predicted the ontreatment platelet reactivity as assessed by VerifyNow P2Y12 assay. The Verigene test offered several advantages for the detection of CYP2C19 polymorphisms such as easiness of use, rapid detection time, and a lower test error rate and test failure rate. We anticipate that the rapid point-of-care CYP2C19 genetic test will clarify the clinical utility of clopidogrel pharmacogenetic tests in patients treated with clopidogrel.

\section{Conflict of Interests}

The authors have no conflict of interests.

\section{Acknowledgments}

This study was supported by a Grant from the Korea Healthcare technology R\&D Project, Ministry for Health, Welfare \& Family Affairs, Republic of Korea (SN: A092258) and by a Grant of the National Project for Personalized Genomic Medicine, Ministry for Health \& Welfare, Republic of Korea (A111218-PG02).

\section{References}

[1] I. Müller, F. Besta, C. Schulz, S. Massberg, A. Schönig, and M. Gawaz, "Prevalence of clopidogrel non-responders among patients with stable angina pectoris scheduled for elective coronary stent placement," Thrombosis and Haemostasis, vol. 89, no. 5, pp. 783-787, 2003.

[2] P. Savi and J. M. Herbert, "Clopidogrel and ticlopidine: P2Y 12 adenosine diphosphate-receptor antagonists for the prevention of atherothrombosis," Seminars in Thrombosis and Hemostasis, vol. 31, no. 2, pp. 174-183, 2005.

[3] B. W. Buchan, J. F. Peterson, C. H. Cogbill et al., "Evaluation of a microarray-based genotyping assay for the rapid detection of cytochrome P450 $2 \mathrm{C} 19^{*} 2$ and ${ }^{*} 3$ polymorphisms from whole 
blood using nanoparticle probes," American Journal of Clinical Pathology, vol. 136, no. 4, pp. 604-608, 2011.

[4] T. Gremmel, S. Steiner, D. Seidinger, R. Koppensteiner, S. Panzer, and C. W. Kopp, "Comparison of methods to evaluate clopidogrel-mediated platelet inhibition after percutaneous intervention with stent implantation," Thrombosis and Haemostasis, vol. 101, no. 2, pp. 333-339, 2009.

[5] L. Bonello, U. S. Tantry, R. Marcucci et al., "Consensus and future directions on the definition of high on-treatment platelet reactivity to adenosine diphosphate," Journal of the American College of Cardiology, vol. 56, no. 12, pp. 919-933, 2010.

[6] L. Chen, S. Qin, J. Xie et al., "Genetic polymorphism analysis of CYP2C19 in Chinese Han populations from different geographic areas of mainland China," Pharmacogenomics, vol. 9, no. 6, pp. 691-702, 2008.

[7] P. Gladding, H. White, J. Voss et al., "Pharmacogenetic testing for clopidogrel using the rapid infiniti analyzer. A DoseEscalation Study," Cardiovascular Interventions, vol. 2, no. 11, pp. 1095-1101, 2009.

[8] C. C. Lee, G. A. McMillin, N. Babic, R. Melis, and K. T. J. Yeo, "Evaluation of a CYP2C19 genotype panel on the GenMark eSensor platform and the comparison to the Autogenomics InfinitiƯ and Luminex CYP2C19 panels," Clinica Chimica Acta, vol. 412, no. 11-12, pp. 1133-1137, 2011.

[9] J. D. Roberts, G. A. Wells, M. R. Le May et al., "Point-ofcare genetic testing for personalisation of antiplatelet treatmetn (RAPID GENE): a prospective, randomised, proof-of-concept trial," The Lancet, vol. 379, no. 9827, pp. 1705-1722, 2012.

[10] M. R. Langley, J. K. Booker, J. P. Evans, H. L. McLeod, and K. E. Weck, "Validation of clinical testing for warfarin sensitivity: comparison of CYP2C9-VKORC1 genotyping assays and warfarin-dosing algorithms," Journal of Molecular Diagnostics, vol. 11, no. 3, pp. 216-225, 2009.

[11] T. A. Nguyen, J. G. Diodati, and C. Pharand, "Resistance to clopidogrel: a review of the evidence," Journal of the American College of Cardiology, vol. 45, no. 8, pp. 1157-1164, 2005.

[12] X. P. Qin, H. G. Xie, W. Wang et al., "Effect of the gene dosage of CgammaP2C19 on diazepam metabolism in Chinese subjects," Clinical Pharmacology and Therapeutics, vol. 66, no. 6, pp. 642646, 1999.

[13] Y. H. Jeong, U. S. Tantry, I. S. Kim et al., "Effect of CYP2C19 ${ }^{*} 2$ and ${ }^{*} 3$ loss-of-function alleles on platelet reactivity and adverse clinical events in East Asian acute myocardial infarction survivors treated with clopidogrel and aspirin," Circulation Cardiovascular Interventions, vol. 4, no. 6, pp. 585-594, 2011.

[14] M. Man, M. Farmen, C. Dumaual et al., "Genetic variation in metabolizing enzyme and transporter genes: comprehensive assessment in 3 major East Asian subpopulations with comparison to Caucasians and Africans," Journal of Clinical Pharmacology, vol. 50, no. 8, pp. 929-940, 2010.

[15] J. J. Storhoff, S. S. Marla, P. Bao et al., "Gold nanoparticle-based detection of genomic DNA targets on microarrays using a novel optical detection system," Biosensors and Bioelectronics, vol. 19, no. 8, pp. 875-883, 2004. 

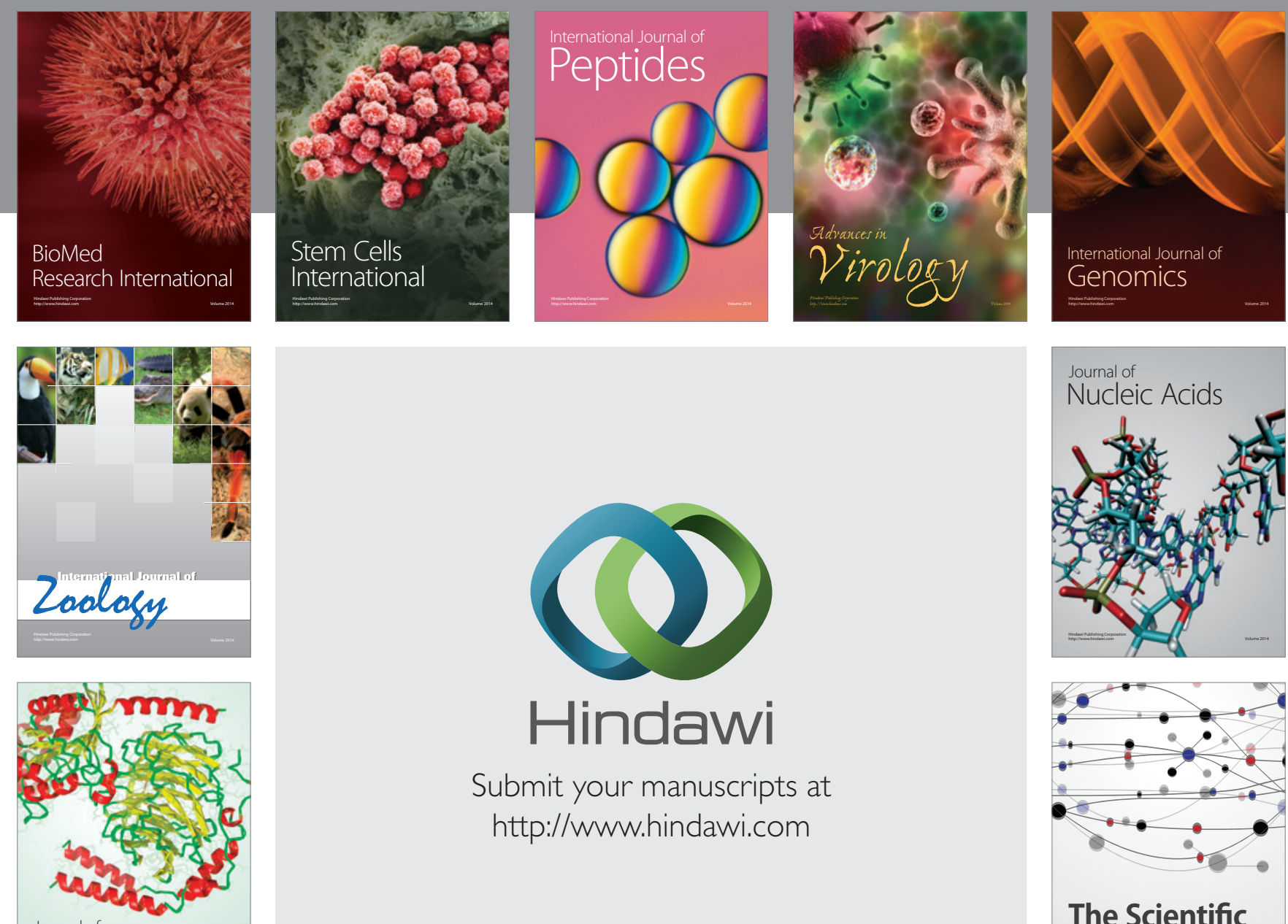

Submit your manuscripts at

http://www.hindawi.com

Journal of
Signal Transduction
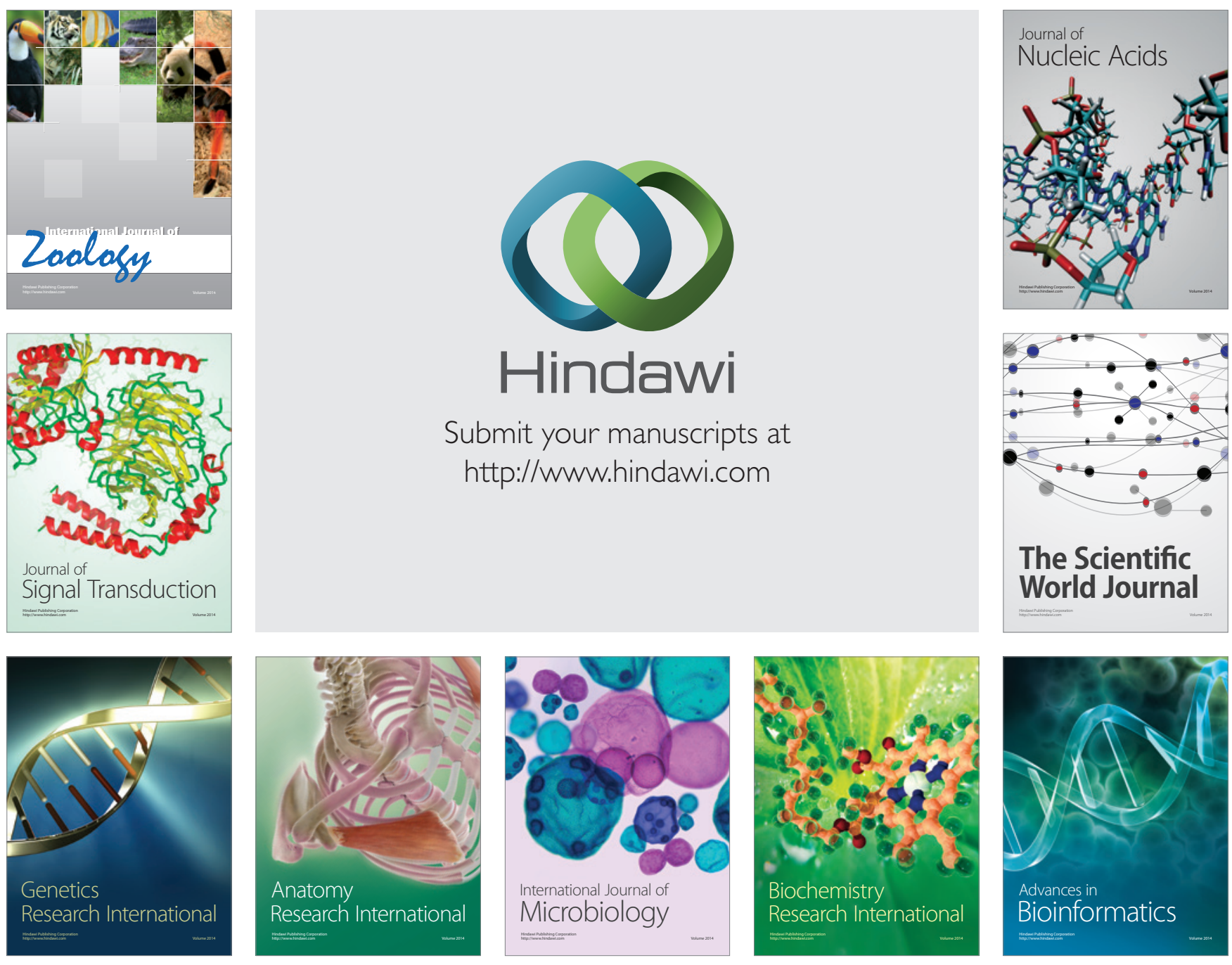

The Scientific World Journal
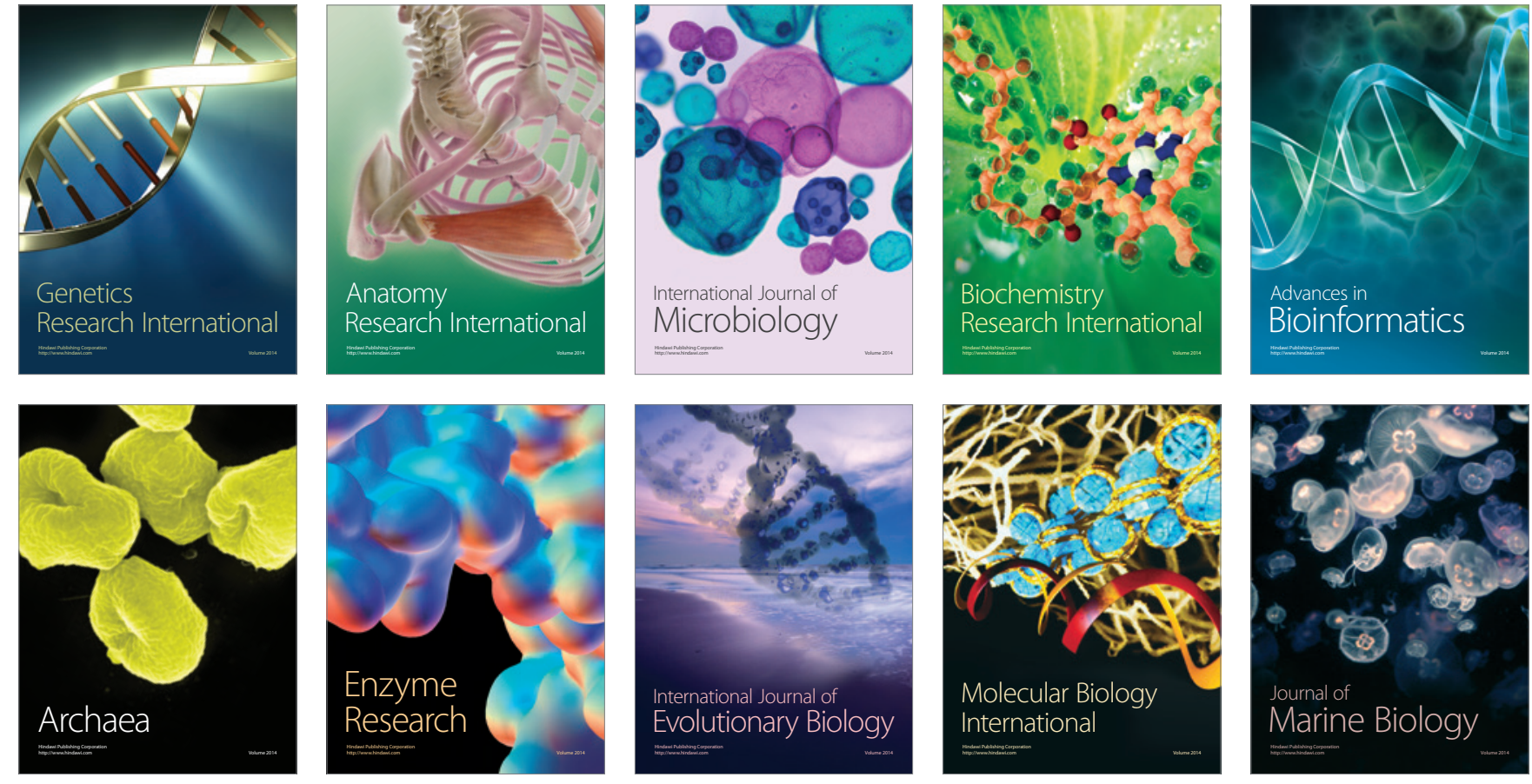